ARTÍCULO ORIGINAL

\title{
Nematodes gastrointestinales en la recría bovina de la región del Chaco Serrano del noroeste de Argentina
}

\author{
Suarez $\mathrm{VH}^{1^{*}}$, Martinez $\mathrm{GM}^{2}$, Viñabal $\mathrm{AE}^{1}$ \\ ${ }^{1}$ INTA - AISA-IIACS Salta, Centro de Investigaciones Agropecuarias \\ ${ }^{2}$ INTA - Estación Experimental Salta
}

* Correspondencia: Victor H. Suarez, INTA Salta, RN 68, km 172, Cerrillos, 4403, Salta, Argentina. E-mail: suarez.victor@inta.gob.ar

Recibido: 26 Abril 2017. Aceptado: 24 Mayo 2017. Disponible en línea: 30 Mayo 2017

Editor: P.M. Beldomenico

RESUMEN. El objetivo del presente trabajo fue estudiar la epidemiología de los nematodes gastrointestinales (NGI) en la recría bovina en la región del Chaco Serrano del Noroeste Argentino durante los períodos mayo-sept. 2014 y mayo-marzo 2015-16. En cada período se formaron tres grupos de 17 terneras de destete: GTS con tratamiento cada 45-50 días con moxidectina, GTL con 1 solo tratamiento: 6/2014 ivermectina y 7/2015 doramectina y GST sin tratamiento. Se realizaron conteos de huevos ( $h p g$ ) y coprocultivos y se registró la ganancia de peso vivo (GPV). Al inicio en ambos períodos los $h p$ fueron moderados $(\bar{X}=515 \pm 449,2014 ; \bar{X}=484 \pm 464$, 2015) elevándose hacia fines del otoño, para luego descender hacia la primavera. Los $h p g$ del GTL mostraron postratamiento solo un descenso parcial y el GTS siempre tuvo valores de $h p g$ muy bajos. Los géneros de nematodes predominantes fueron Cooperia (41,8\%), y Haemonchus (53,7\%). En ambos períodos el efecto de los tratamientos se evidenció desde el invierno, mostrando el GTS una mayor GPV $(p<0,05)$ que la de los otros dos grupos cuando se mejoró la oferta alimenticia. Las GPV totales del GTS fueron mayores. Al final del ensayo, los promedios en la GPV fueron en $\mathrm{kg}$ de $11,2,11,5$ y 19,6 (2014) y de $54,3,56,5$ y 73,4 (2015-16) respectivamente para el GST, GTL y el GTS.

SUMMARY. Gastrointestinal nematode infection on beef cattle heifers in the Chaco Serrano region of northwestern Argentina. The aim of this work was to study the epidemiology of gastrointestinal nematodes (GIN) on beef cattle in the Chaco Serrano region of northwestern Argentina, during the periods May-Sept. 2014 and May -March 2015-16. During each period, three groups of 17 heifer calves were performed: STG: calves treated every 45-50 days with moxidectin, LTG: calves treated once, June/2014 ivermectin and July/2015 doramectin and UTG: untreated calves. Eggs per gram of feces (epg) and feces cultures were performed. Live weight gain (LWG) was recorded. At the start of both periods the mean calf epg was moderate $(\bar{X}=515 \pm 449,2014 ; \bar{X}=484 \pm 464$, 2015) and peaks towards late autumn, to decrease forward the spring. LTG epg post treatment only decreases partially and STG epg always showed negligible values. Predominant genera were Cooperia (41.8\%) and Haemonchus (53.7\%). In both periods, the treatment effect was evident from the winter, showing STG a significant $(p<0.05)$ higher LWG than the other groups when the ranch increased the alimentary offer. At the end of the trial, average total LWG were in $\mathrm{kg}, 11.2,11.5$ and 19.6 (2014) and $54.3,56.5$ and 73.4 (2015) respectively for NTG, LTG y el STG.

Palabras clave: bovinos; nematodes gastrointestinales; epidemiología; efecto; noroeste argentino.

Key words: cattle, gastrointestinal nematodes, epidemiology; effect; northwestern Argentina.

\section{Introducción}

La producción ganadera del NOA, especialmente la cría bovina, cobra día a día más importancia, por el potencial aporte que puede brindar al sector. Así, podemos ver cómo en la región, del 2003 al 2010 las existencias han crecido en gran medida, en Salta, de 562.252 a 1.049.782 cabezas, mientras que en Santiago del Es- tero de 1.157 .779 a 1.397 .580 cabezas (Milano, 2011). Este crecimiento en áreas subtropicales, con poca experiencia en cría bovina de tipo intensivo, ha llevado a los productores ganaderos a confrontar con limitantes productivas como problemáticas que afectan la salud del ganado.

Una de esas limitantes que hacen a la salud del ganado, la constituyen las infestaciones por nematodes gastrointestinales (NGI), las cuales pueden afectar la productividad de los bovinos (Armour, 1980; Barger, 1993; Fiel et al., 2013; Suarez et al, 2013). Los NGI presentan una estrecha relación entre las formas de 
vida libre y los factores climáticos y el manejo animal, afectando su desarrollo y supervivencia (Armour, 1980; Suarez y Lorenzo, 2000). Esto lleva a encontrar marcadas diferencias regionales entre la epidemiología y el efecto sobre la producción bovina que pueden causar los NGI.

En el noroeste argentino (NOA), la región chaqueña semiárida se caracteriza por un clima subtropical, caracterizado por una estación de lluvias estivales y una estación seca que se prolonga de abril a noviembre. Esta región a su vez puede dividirse en lo que se denomina Chaco Serrano y Chaco semiárido. El Chaco Serrano, es una zona agroecológica, ubicada entre serranías, la cual se presenta muy modificada por la actividad ganadera, que por lo general se realiza sobre pasturas naturales o implantadas (Bianchi, 1992).

Los antecedentes sobre el estudio de los parásitos internos que parasitan a los bovinos en el NOA son escasos, más si se pretende abarcar la diversidad de ambientes que esta región posee (Le Riche et al., 1982; Kühne et al., 1986; Suárez et al., 2016). En particular esta región del Chaco Serrano posee muy pocos estudios previos sobre la epidemiología y efectos de los NGI sobre el ganado de cría bovino.

Debido a este vacío de información, el objetivo del presente ensayo fue estudiar la epidemiología y los efectos de los NGI en las terneras de reposición en una región representativa del Chaco Serrano en Salta.

\section{Materiales y Métodos}

El estudio se llevó a cabo en un establecimiento de cría bovina, ubicado en el Valle de Sianca, Dto de Gral. Güemes, Salta, según punto de GPS $24^{\circ} 37^{\prime} 10^{\prime \prime} S$ y $64^{\circ} 47^{\prime} 28^{\prime \prime} \mathrm{O}$ y a $742 \mathrm{msnm}$. La región tiene un régimen de lluvias estivales muy irregular que promedian los 480 (extremos: 300-600) mm anuales y que se interrumpe con un período seco que se prolonga de abril a octubre (Bianchi, 1992).

El lote en estudio estuvo compuesto por terneras cruza Braford, destetadas precozmente en mayo a los 4-5 meses de edad. La oferta alimenticia fue insuficiente, basándose en grano de maíz, alimento balanceado, heno de alfalfa y o silo de maíz suministrados en potreros degradados con gatton panic (Megathyrsus maximus), o buffel grass (Cenchrus ciliaris) y malezas, a razón de entre 30 y 120 terneras por $\mathrm{Ha}$, por períodos de 15 a 60 días de acuerdo a la carga animal.

Durante los períodos $14 / 5 / 14$ al $25 / 9 / 14$ y $26 / 5 / 15$ al 2/3/16 se monitorearon 51 terneras de destete naturalmente infestadas por NGI. Con tal fin, se formaron en cada período tres grupos de 17 terneras cada uno, para comparar una posible respuesta frente a los tra- tamientos antihelmínticos y el efecto de los parásitos sobre la ganancia de peso vivo. Los grupos en ambos períodos fueron: grupo sin tratamiento (GST); grupo con tratamiento sistemático al momento de cada muestreo cada 45-50 días (GTS), con moxidectina (MXD) subcutánea a razón de $200 \mu \mathrm{g} / \mathrm{kg}$; grupo sujeto al tratamiento implementado localmente de acuerdo al manejo sanitario del establecimiento (GTL). Este grupo fue tratado con ivermectina (IVM) y doramectina (DRM), ambas a razón de $200 \mu \mathrm{g} / \mathrm{kg}$, respectivamente el $4 / 6 / 14$ y el $30 / 7 / 15$. Los grupos pastorearon juntos con todo el lote, compuesto además por 100-150 terneras de recría.

Cada 45-50 días se tomaron muestras individuales de heces de las terneras para realizar conteos individuales de huevos de nematodes ( $h p g$ ) por la técnica de McMaster modificada (Roberts y O'Sullivan, 1949). Además se diferenciaron los géneros de NGI (Keith, 1953) de pooles provenientes de cada grupo como también se procedió con la técnica de Baermann para recuperar larvas de vermes pulmonares (Suarez, 1997). Asimismo se realizó el diagnóstico de Fasciola de pooles mediante el método de sedimentación y coloración de azul de metileno descripto por Viñabal et al. (2015). También se llevó a cabo una prueba de reducción del conteo de huevos de NGI pos tratamiento del GTL para ver la eficacia de las avermectinas y el MXD (Coles et al., 1992).

La ganancia de peso vivo (GPV) se evaluó mediante pesadas mensuales de los bovinos con desbaste previo de 18 horas.

Las diferencias entre la ganancia de peso y los conteos de huevos $(h p g)$ se compararon por análisis de varianza y las diferencias entre grupos se realizaron mediante prueba de Tukey. Los valores de los hpg se normalizaron mediante transformación logarítmica $(\log x)$. Para los análisis se contó con el programa INFO STAT (Di Rienzo et al., 2008).

\section{Resultados}

Las temperaturas medias y precipitaciones mensuales acontecidas durante el ensayo se muestran en la Figura 1. Las temperaturas medias mensuales para los meses más extremos fueron en cuanto a temperatura máxima y mínima respectivamente para diciembre de 40,7 y $13,8{ }^{\circ} \mathrm{C}$ y para julio de 28,6 y $1,0^{\circ} \mathrm{C}$. Las precipitaciones máximas mensuales que se registraron durante el período 2014 sumaron $492 \mathrm{~mm}$, pero durante el periodo de ensayo solo se registró $14,8 \mathrm{~mm}$ y durante el período de ensayo del 2015-16 las precipitaciones alcanzaron los $356 \mathrm{~mm}$, aunque de ese total, la mayor parte $(312 \mathrm{~mm}$ ) cayó durante enerofebrero del 2016. La Figura 2 presenta los $h p g$ de los dos períodos. 


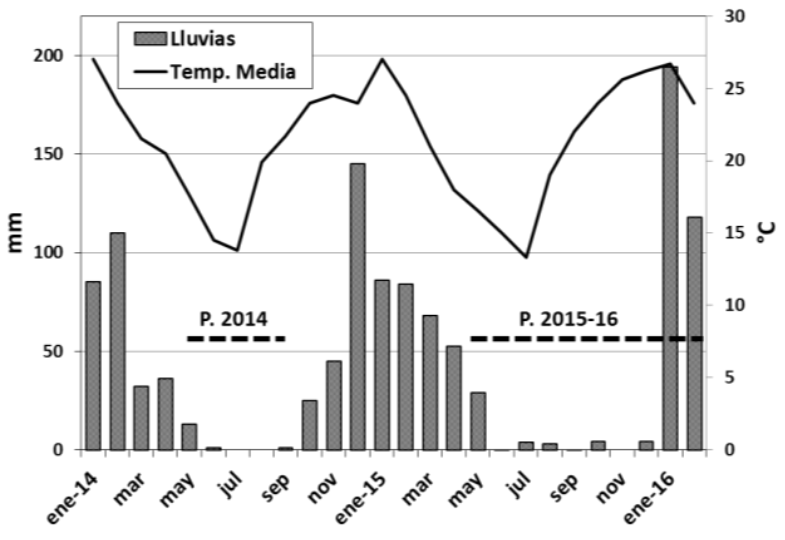

Figura 1. Temperaturas medias y precipitaciones mensuales registradas durante el período de ensayo

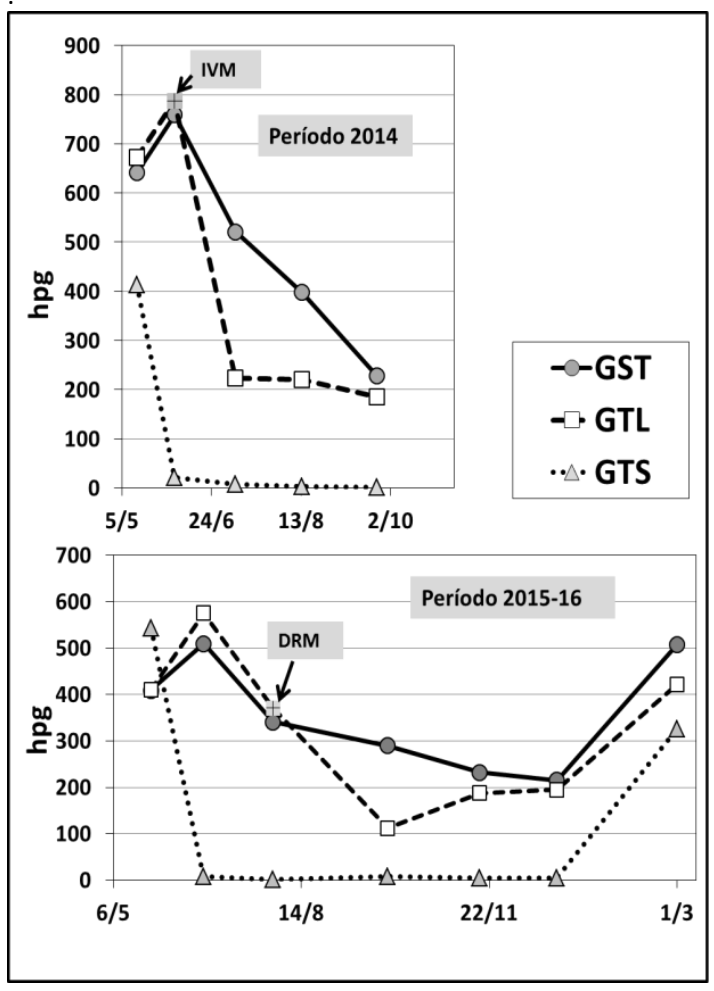

Figura 2. Media de los conteos de huevos (hpg) de la recría durante los períodos 2014 y 2015-16. GTS (Tratamiento sistemático), GTL (tratamiento local) y GST (sin tratamiento). IVM: tratamiento con ivermectina; DRM: tratamiento con doramectina.

Al inicio en ambos períodos los hpg promedios de los grupos GST fueron moderados, elevándose hacia fines de otoño. En mayo 2014 los conteos en promedio dieron $515 \pm 449 \mathrm{hpg}$, para elevarse al 4/6/14 a 759 $\pm 615 \mathrm{hpg}$, lo que motivó el tratamiento del GTL con IVM. Durante el 2015 los conteos iniciales promedia- ron $484 \pm 464 \mathrm{hpg}$, elevándose hacia fines de junio (GST: $585 \pm 476 \mathrm{hpg}$ ), motivando en el GTL el tratamiento con DRM a fines de julio. Posteriormente, los hpg descendieron hasta la primavera en ambos períodos, para subir en el período 2015-16 a partir de enero hasta el final de las observaciones.

El grupo GTS siempre tuvo valores de $h p g$ muy bajos, mostrando una eficacia de la MXD del 95\%. Los $h p g$ del GTL mostraron pos tratamiento solo un descenso parcial, siendo la eficacia de la IVM del $71,6 \%$ y de la DRM del 73,0\%.

En cuanto a la recuperación por coprocultivos del GST de los géneros de $\mathrm{NGI}$, predominaron en promedio en ambos períodos Haemonchus $(53,7 \%)$ y Cooperia $(41,8 \%)$; también en forma escasa se recuperaron larvas de Oesophagostomum (4,2\%) y Trichostrongylus $(0,23 \%)$. La Figura 3 señala que a partir del otoño, se observa la presencia de los géneros Haemonchus, y en menor medida Cooperia para luego esta última elevarse en invierno hasta septiembre donde la presencia de Haemonchus prevalece nuevamente. Hacia marzo en terneras mayores de un año los porcentajes de Haemonchus y Cooperia se igualan. La presencia de Oesophagostomum fue mayor en otoño.

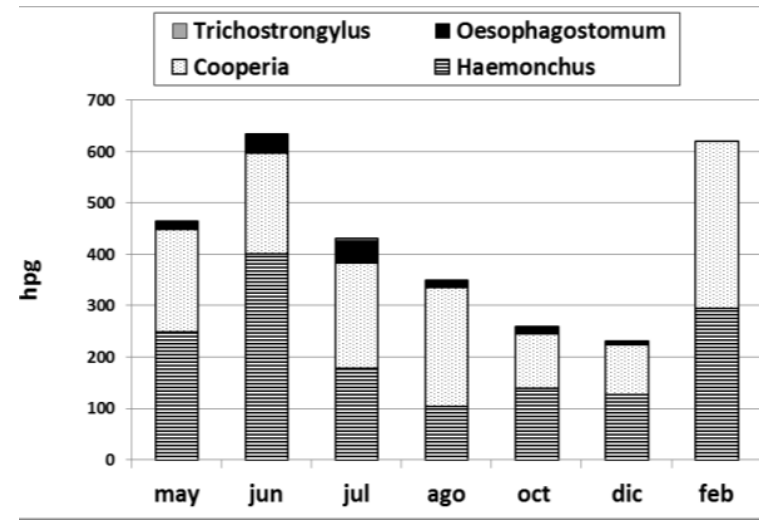

Figura 3. Promedio de la proporción de los géneros de nematodes recuperados de los coprocultivos según el $h p g$ promedio durante todo el estudio.

La proporción de géneros recuperadas del GTL fue similar a la descripta para el GST, aunque luego de los tratamientos antiparasitarios el género prevalente fue Cooperia, $(72,5 \%)$ y en menor medida Haemonchus, 27,5\%. En el caso del GTS, también Cooperia fue el género prevalente (Cooperia: $87 \%$, Haemonchus: $12 \%$, Oesophagostomum: $1 \%)$.

Se recuperaron huevos de Fasciola hepatica en bajo número en mayo, junio y septiembre del 2014 y junio del 2015. No se recuperaron larvas de Dictyocaulus. 
Durante todo el ensayo no se observaron en las terneras signos clínicos de gastroenteritis verminosa, ni hubo signos de otros problemas sanitarios en la tropa.

A lo largo del período 2014 no se observaron diferencias en la GPV de los grupos hasta agosto, cuando se registró una GPV superior $(p<0,05)$ en el GTS con respecto a la del GST, quedando el GTL con una GPV intermedia entre ambos. Las diferencias $(p=0,046)$ se ampliaron en el GTS sobre los otros 2 grupos hacia el final de las observaciones del 2014 (Figura 4), resultando las GPV de 19,6, 11,5 y 11,2 kg para GTS, GTL y GST respectivamente.

Durante el período 2015-16, desde mayo a noviembre el GTS mostró GPV $(26,0 \mathrm{~kg})$ más elevadas $(p=0,045)$ que las del GTL $(22,7 \mathrm{~kg})$ y las del GST $(17,9 \mathrm{~kg})$. Posteriormente hasta la pesada final del 2 de marzo, las ganancias se diferenciaron algo más entre grupos, resultando la GPV promedio del GTS $(73,4 \mathrm{~kg})$ mayor $(p=0,02)$ a la del GTL $(56,5)$ y del GST $(54,3 \mathrm{~kg})$, que no difirieron entre ellas. El GTS logró un incremento en la GPV del $31,1 \%$ y del $35,1 \%$ sobre la del GTL y del GST respectivamente (Figura 4).

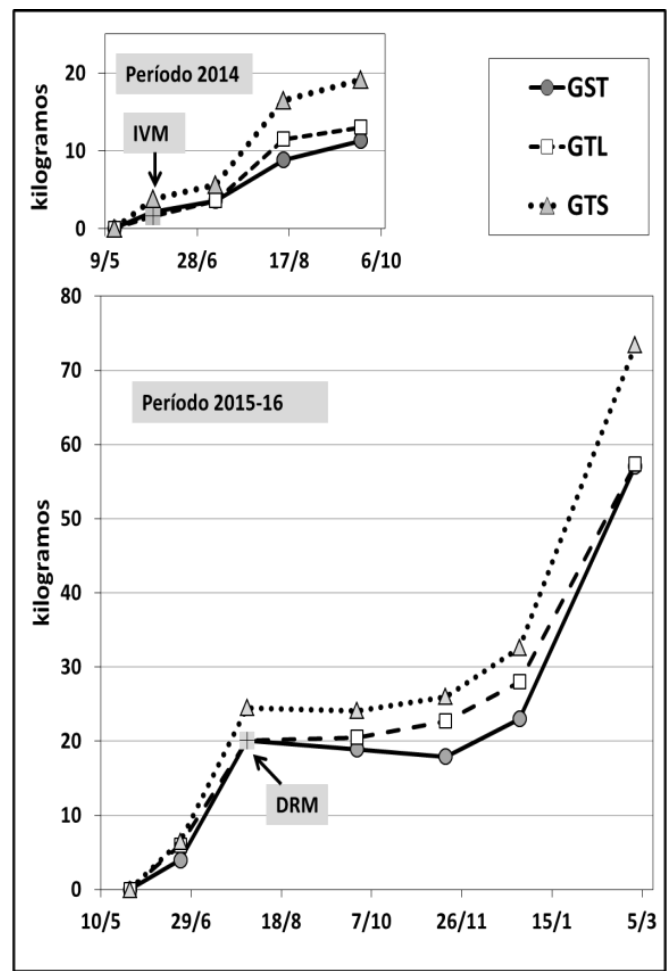

Figura 4. Ganancias de peso vivo en kg de las terneras en los periodos 2014 y 2015-16. GST: grupo sin tratamiento; GTS grupo con tratamiento sistemático con moxidectina; GTL grupo con el tratamiento del establecimiento.+ IVM: tratamiento con ivermectina; + DRM: tratamiento con doramectina.

\section{Discusión}

Las temperaturas fueron similares a los promedios históricos al igual que la distribución estival de las lluvias, aunque durante el 2015, luego de un otoño húmedo, las precipitaciones estuvieron por debajo de la media histórica, sobre todo en lo referente a los meses de noviembre y diciembre. Los sucesos meteorológicos acontecidos durante el ensayo se relacionaron con la irregularidad del clima en esta región, otorgándole a estos resultados validez epidemiológica, debido a la estrecha relación entre los NGI y el clima.

Los resultados parasitológicos muestran que a mediados de otoño, los hpg fueron de moderados a altos (759 / 585), dependiendo estos valores de la infestación soportada a partir del destete precoz a mediados del verano. Esta elevación de los hpg hacia el final del otoño, fue similar a lo observado por Kühne et al. (1986) en la región chaco salteño semiárido. Por el contrario, estos resultados discrepan de aquellos observados en el área pampeana central y en la región del pastizal serrano del NOA (Suarez et al, 1999; Suarez et al, 2013; Suarez y Viñabal, 2016), donde el pico del $h p g$ se describe a fines de otoño o mediados de invierno. Observaciones realizadas en el oeste pampeano evidenciaron que el pie de infestación de las vacas o de los animales de sobreaño de fin de verano o de inicios de otoño se mantiene gracias a la humedad ambiente y a las lluvias (Suarez y Lorenzo, 2000). Muestreos realizados a fines de febrero en este mismo establecimiento sobre terneros con destete precoz de enero y suplementados sobre piquetes empastados, muestran promedios de $h p g$ elevados ( $h p g=1189$ y $80 \%$ de Haemonchus), indicando que este destete a una edad muy joven, sin una desparasitación previa a la entrada a pastoreo, sería una fuente importante de contaminación otoñal de los potreros. También este pico descripto en los hpg, tendría su origen en la mayor presencia de Haemonchus en otoño.

Luego los hpg del GST comenzaron a descender desde el inicio del invierno hacia la primavera, con una disminución del género Haemonchus, en favor de un aumento de Cooperia, probablemente a partir de la pobre disponibilidad de larvas en los potreros debido a la escasez de lluvias inverno primaverales (Suarez, 2001). Esta tendencia en los hpg, también observada en la recría de región de selvas y pastizales pedemontanos (Suarez et al., 2016), muestra el forta- 
lecimiento de la inmunidad de las terneras de año frente a los NGI.

Los hpg del GTL mostraron postratamiento solo un descenso parcial, siendo la eficacia de la IVM del $72 \%$ (45\% para Cooperia) lo cual al compararlo con la eficacia de la MXD (95\%) sugiere la existencia de resistencia antihelmíntica (RA). Este hecho, debido a la falta de antecedentes de resistencia antihelmíntica en el NOA, señala que la presencia de resistencia debería estudiarse. El hecho de los frecuentes tratamientos con IVM en el NOA para combatir las garrapatas sería la causa más probable de RA frente a las avermectinas (Suarez y Cristel, 2014).

Con respecto a la presencia de $F$. hepatica, se recuperaron huevos esporádicamente en otoño e invierno, solo cuando las terneras pastorearon alfalfa bajo riego, evidenciando la distribución de este trematode a partir de las acequias y del riego por inundación.

Los resultados productivos en los dos períodos de estudio, muestran que en determinados momentos no hubo respuestas pos-tratamiento en las ganancias de peso del grupo GTS, a pesar de la caída de los $h p g$. Esta falta de respuesta probablemente se deba a la pobre oferta nutricional a que en general fueron sometidos los terneros a lo largo de los dos períodos de ensayo, oferta que no les permitió expresar una mejor GPV acorde a una buena alimentación. Esto puede probarse, ya que las mayores diferencias en la GPV pos-tratamiento en el período 2014 se registraron a mediados del invierno cuando las terneras ingresaron a una pastura de alfalfa bajo riego suplementadas con grano y silo de maíz. Durante el período 2015-16, las terneras desde mediados de junio hasta el rebrote del pasto de fines de primavera estuvieron en pasturas de muy pobre calidad, lo que se relaciona con la lenta respuesta en la GPV a los tratamientos. Posiblemente las respuestas posteriores a las desparasitaciones en ambos períodos se deban a la carga parasitaria residual que los terneros del GST padecían desde mediados de otoño, ya que la falta de lluvias invernales no habría favorecido la contaminación de los potreros. Ensayos realizados en el INTA Anguil, La Pampa, muestran un efecto nocivo de las infestaciones mixtas preexistes sobre la GPV del grupo no tratado del $20 \%$ en comparación con los tratados en los primeros 70 días de ensayo, a pesar de haber pastoreado verdeos limpios de larvas, sin posibilidades de reinfestación (Suarez y Cristel, 2005).

A diferencia de lo que ocurre en la región central del país, donde Ostertagia ostertagi, Trichostrongylus axei, Cooperia oncophora y Haemonchus placei son los nematodes que afectan la productividad del ganado (Suarez, 1990), en el presente ensayo no se halló el género Ostertagia y los vermes involucrados estuvieron representados por Haemonchus placei y Cooperia pectinata y $C$. punctata. Esta última especie no está citada en el NOA y aunque ha sido identificada en el presente estudio a partir de la recuperación de larvas infestantes de coprocultivos, hay evidencia de su presencia a partir de la recuperación de formas adultas de $C$. punctata y $C$. pectinata de necropsias realizadas en la región (Suarez, datos no publicados). En el caso de estas últimas especies, trabajos brasileños consideran a $C$. punctata y $C$. pectinata más patógenas que $C$. oncophora (Guimaraes et al., 1990). A este respecto, algunas citas describen los efectos de estas especies de Cooperia sobre los terneros, con anorexia, pérdida de proteínas plasmáticas, retraso en el crecimiento, heces blandas, y a la necropsia engrosamiento de las vellosidades intestinales y producción excesiva de moco (Armour et al., 1987; Stromberg et al., 2012).

Por otro lado, es interesante señalar la falta de GPV compensatoria del GST durante el verano 2016 en relación con el GTS, lapso en el cual se lo dejó de tratar por 62 días, bajo una oferta forrajera que cubría bien los requerimientos de los animales. Este fenómeno fue comunicado previamente en infestaciones debidas principalmente a Ostertagia (Suarez et al., 1991; Suarez y Cristel, 2005; Fiel et al., 2013) y recientemente en el norte de Salta, con infestaciones donde predominaban Cooperia y Haemonchus (Suarez et al., 2016).

A partir de estos resultados se puede concluir que los géneros Cooperia y Haemonchus fueron los NGI prevalentes, aunque Oesophagostomum y Trichostrongylus también estuvieron presentes a lo largo de las observaciones. La mayor incidencia de los NGI sobre la recría se evidenció a través de los hpg, los cuales mostraron un pico hacia el otoño.

Las diferencias en la GPV entre grupos en respuesta a los tratamientos a pesar de la pobre oferta forrajera $y$ la reducida eficacia de las avermectinas, evidencian el impacto de los NGI sobre la recría bovina y señalan la necesidad de estudiar las estrategias de control adecuadas a los diferentes sistemas productivos y la presencia de resistencia antihelmíntica.

\section{Agradecimientos}


Los autores agradecen al Sr Felipe Badía y a la empresa El Monerío S.A. por la hospitalidad brindada y la oportunidad de trabajar en su establecimiento.

\section{Bibliografía}

Armour J. 1980. The epidemiology of helminth disease in farm animals. Vet. Parasitol. 6: 7-46.

Armour J, Bairden K, Holmes PH, Parkins JJ, Ploeger H, Salman SK, McWilliam PN. 1987. Pathophysiological and parasitological studies on Cooperia oncophora infections in calves. Res. Vet. Sci. 42: 373-381.

Barger IA. 1993. Helminth parasites and animal production. Symposium of Parasitology, University of Sydney, Australia pp. 133-155.

Bianchi A. 1992. Regiones productivas de Salta y Jujuy. Rev. Panorama Agropecuario $N^{\circ} 41$, pp. 4-14.

Coles GC, Bauer C, Borgsteede FH, Geerts S, Klei TR, Taylor MA, Waller PJ. 1992. World Association for the Advancement of Veterinary Parasitology (WAAVP), methods for the detection of anthelmintic resistance in nematodes of veterinary importance. Vet. Parasitol. 44: 35-44.

Di Rienzo JA, Casanoves F, Balzarini MG, Gonzalez L, Tablada M, Robledo CW. 2008. InfoStat, versión 2008, Grupo InfoStat, FCA, Universidad Nacional de Córdoba, Argentina.

Fiel C, Steffan P, Entrocasso C. 2013. Epidemiologia e impacto productivo de nematodos en la Pampa Húmeda. (Cap. 2. pp. 29-58). En: Fiel C, Nari A (eds.). Enfermedades parasitarias de importancia clínica y productiva en rumiantes. Fundamentos epidemiológicos para su diagnóstico y control. Editorial Hemisferio Sur.

Guimaraes MP, Costa HM, Lima WS. 1990. Prevalence of Cooperia punctata, $C$. pectinata and $C$. oncophora infections in dairy calves in Brazil. J. Helminthol. 64: 319-322.

Keith RK. 1953. The differentiation of the infective larvae of some common nematode parasites of cattle. Aust.J. Zool. 1 223-235.

Kühne GI, Le Riche PD, Dwinger RH. 1986. Parasitismo gastrointestinal en bovinos en cuatro zonas ecológicas de la provincia de Salta. Rev. Med. Vet. (B. Aires) 67: 128-136.

Le Riche PD, Kühne GI, Dwinger RH. 1982. An epidemiological study of helminthiasis in cattle in subtropical Argentina. Trop. Anim. Health Prod. 14: 207-215.

Milano R. 2011. El nuevo escenario de la ganadería argentina. Revista de la Bolsa de Comercio de Rosario, año 100, n. 1514, sept 2011, pp. 24-30.

Roberts F y O'Sullivan P. 1949. Methods for egg counts and larval cultures for strongyles infecting the gastrointestinal tract of cattle. Aust. J. Agric. Res. 1: 99-103.

Stromberg BE, Gasbarre LC, Waitec A, Bechtol DT, Brownd MS, Robinsona NA, Olsona EJ, Newcombe H. 2012. Cooperia punctata: Effect on cattle productivity? Vet. Parasitol. 183: 284-291

Suarez VH. 1990. Inhibition patterns and seasonal availability of nematode for beef cattle grazing on Argentina's Western Pampas. Int. J. Parasitol. 20: 10311036.
Suarez VH. 1997. Diagnóstico de las parasitosis internas de los rumiantes en la región de invernada. Técnicas e Interpretación. Bol. Divulgación Técnica (INTA-Anguil) 56, 50 p.

Suarez VH. 2001. Ecología de los estadios de vida libre de los nematodes bovinos durante la contaminación otoño invernal en la región semiárida pampeana. Rev. Med. Vet. (B. Aires) 82: 316-323

Suarez VH, Bedotti, DO, Larrea S, Busetti MR, Garriz CA.1991. Effect of an integrated control programme with ivermectin on growth carcase and nematode infection on beef cattle in Argentina's Western Pampas. Res. Vet. Sci. 50: 195199.

Suarez VH, Lorenzo R.M., Busetti M.R, Santucho GM. 1999. Physiological and parasitological responses to nematode infections of fattening cattle in the western pampas of Argentina. Vet. Parasitol. 81: 137-148

Suarez VH, Lorenzo RM. 2000. Ecology of the free living stages of cattle nematodes during estival contamination in Argentina western pampas. Parasite (Fr), 7: 255-261.

Suarez VH, Cristel SL. 2005. Mínimo uso de antihelmínticos y manejo forrajero en el control de los nematodes bovinos en la región semiárida pampeana. (pp. 95-102). En: Resistencia a los antiparasitarios internos en la Argentina, FAO/TCP, Argentina.

Suarez VH, Rossanigo CE, Descarga C. 2013. Epidemiologia e impacto productivo de nematodos en la Pampa Central de Argentina. (Cap. 3. pp. 59-88). En: Fiel C, Nari A (eds.). Enfermedades parasitarias de importancia clínica y productiva en rumiantes. Fundamentos epidemiológicos para su diagnóstico y control. Editorial Hemisferio Sur.

Suarez VH, Cristel, SL. 2014. Risk factors for anthelmintic resistance development in cattle gastrointestinal nematodes in Argentina. Braz. J. Vet. Parasitol., 23: 129-135.

Suarez VH, Martínez GM, Micheloud JF, Viñabal AE. 2016 Epidemiología y efecto de los parásitos gastrointestinales en la cría bovina de la región de selvas y pastizales pedemontanos. XXI Reunión Científico Técnica Asociación Arg. Veterinarios Laboratorios de Diagnóstico S.S. Jujuy, 6-8 oct-2016, Sección P 20.

Suarez VH y Viñabal AE 2016. Epidemiología y efecto de los nematodes gastrointestinales en bovinos de carne en la región del pastizal serrano del noroeste argentino. Rev. Arg. Prod. Anim. 36: 57

ViñabaL AE, Cafrune MM, Aguirre DH, Bassanetti AF, Bertoni EA, Suarez VH. 2015. Propuesta y evaluación de una técnica de sedimentación y tinción con Azul de Metileno (y de una variante) para el diagnóstico de Fasciola hepatica. Rev. Vet. Arg. 327: 1-11 